RECENSIONES DE TESIS 



\title{
LA REGULACIÓN DE \\ LAS TARIFAS EN EL \\ SECTOR SANITARIO DE LA \\ PROVINCIA DE SANTA FE
}

\author{
Tesista: \\ Eduardo Luis Visentini
}

Director de Tesis:

Dr. Miguel Angel Asensio

1. MARCO TEÓRICO:

\section{A PARTIR DE LA DÉCADA DE LOS ' 90}

A fines de la década de los '80 y durante los ' 90 ante la profunda crisis desatada, la Argentina se vio forzada a llevar a cabo una serie de importantes transformaciones económicas, sociales y culturales, que fueron desarmando el viejo aparato estatal.

Aquel Estado protector, popular-social que comienza a dar sus primeros pasos en nuestro país en la década de los '30 empieza a retirarse, retrocediendo ante el fuerte auge de las "políticas Neoliberales" que abren importantes procesos de privatizaciones, donde, el Estado convierte sus aspectos funcionales de productor y prestador de servicios, en regulador y controlador de los servicios públicos.

El descrédito generalizado en las empresas públicas, el contexto económico caótico signado por la hiperinflación, el alto nivel de déficit fiscal y la necesidad de iniciar un proce- so de reinversión en los Organismo estatales modernizando sus estructuras productivas, puso en marcha el proceso privatizador de los años ' 90.

A partir de este momento, la actividad pública comienza a transformarse, orientándose hacia un rol "mínimo", traducido en un debilitamiento de la autoridad del Estado Central, descentralización, retiro y desmantelamiento, vinculado a una profunda eliminación de las funciones empresariales y productivas. En su metamorfosis, el Estado "Elefantiásico" o Empresario que tenía las funciones productivas y de comercialización, junto a funciones de control y fiscalización, comienza a transformarse, desprendiéndose de las primeras (producción y comercialización) para controlar y regular el mercado. Este nuevo Estado tiene otras funciones, como la de establecer los ré- 
gimen de explotación de los servicios públicos, fiscalización del cumplimiento de las normas relativas a la prestación de los servicios sanitarios, régimen tarifarios, régimen de subsidios, reglamento del usuario, régimen de sanciones por incumplimientos, etc.

Como se sabe, economistas han protaginozado prolongados debates doctrinarios acerca de la perfomance en la provisión de los servicios públicos, centrando su discusión en torno a si los mismos deben estar en manos privadas o estatales. En esa línea la experiencia empírica muestra una creciente conveniencia hacia la provisión de los servicios en la órbita privada. Con relación a ello, Kessides (1993) muestra las ventajas que trae aparejadas la participación privada en la provisión de los mismos, tales como: habilidades en la dirección, eficiencia productiva, eficiencia dinámica, satisfacción al cliente y autonomía financiera.

Sin embargo, para la determinación pública o privada en la provisión de los servicios se debe tener en cuenta el bien a proveer, evaluando o ponderando variables tales como, bien de consumo rival o conjunto, existencia de externalidades, generación de economías de escalas, costos hundidos, etc.

Se pueden encontrar diversas causas del fracaso estatal en la provisión de los servicios en la Argentina, así la inclusión de objetivos múltiples exigidos a las empresas públicas, ausencia de incentivos para mejorar la performance, insuficiencias presupuestarias en algunos casos, conductas oportunistas de los directivos en las empresas públicas, fijación de precios e inversiones inconsistente a los niveles reales, no implementación de auditorias de desempeño, fueron solo algunos de los motivos en que el Gobierno decidiera la transformación de los servicios público en el país.

Este conjunto de causas, sumados a los paradigmas existentes en los mercados internacionales con respecto a la incorporación y aplicación de políticas liberales, minaron la posibilidad de continuar con un importante universo de empresas públicas que se desenvolvieron hasta fines de los '80.

Sin embargo a través del creciente interés académico en los problemas planteados por las privatizaciones o concesiones de los servicios públicos, hay quienes cuestionan la implementación de éstas políticas en los monopolios naturales (caso concreto suministro de agua potable y desagües cloacales), argumentando que en lugar de desprenderse de la prestación de estos servicios se debieran haber producido los cambios de propiedad en aquellas industrias que potencialmente estaban en condiciones de operar en entornos competitivos (caso Energía eléctrica, telecomunicaciones, transporte, etc.).

\section{LA TRANSFORMACIÓN DEL SECTOR SA- NITARIO DE LA PROV INCIA DE SANTA FE}

Los servicios sanitarios en la Argentina inicialmente estuvieron en manos del Estado Nacional. La antigua Empresa "Obras Sanitarias de la Nación" era la encargada de brindar el suministro de agua potable y servicios Cloacales desde 1912. A partir del $1^{\circ}$ de agosto de 1980 los servicios pasan a la órbita del Estado Provincial, creándose mediante la Ley 8711 la "Dirección Provincial de Obras Sanitarias" (DIPOS), Organismo cuya dependencia funcional recaía en el Ministerio de Obras, Servicios Públicos y Vivienda. Su finalidad se enmarcaba en el estudio, proyección, conducción, ampliación y explotación de las obras de provisión de agua y evacuación de efluentes Cloacales y residuales, como así también el suministro de agua por interconexiones mediante el sistema de acueducto. A tales propósitos le correspondía la exploración, alumbramiento y utilización de las aguas subterráneas, y el control de la calidad y nivel de contaminación del agua de bebida y efluentes (art. $3^{\circ}$ - Ley 8711/1980).

El Organismo se caracterizaba por estar 
altamente condicionado a disposiciones ministeriales centralizadas, con escasa toma de decisiones por parte de los directivos, con profundas restricciones presupuestarias. A pesar de que el servicio tenía autofinanciamiento, se priorizaban inversiones selectivas en infraestructura (provocando una marcada desinversión en el sector), sin considerar aspectos de eficiencia. Sumado a esta situación, el clientelismo político y sindical en la incorporación a su planta de personal, hacían del mismo un objeto diario de abusos casi intolerables, instituyendo a la Organización en una pesada maquinaria burocrática con poca cintura gimnástica para la gestión, lo que la transformó en candidata para protagonizar el proceso privatizador en la Provincia de Santa $\mathrm{Fe}$, siendo paradójicamente junto a la Empresa de Energía las únicas organizaciones provinciales en que primero se orientaron las miradas para la transferencia de sus servicios a manos privadas a pesar de que financieramente siempre tuvieron una aceptable perfomance.

Con la promulgación de la Ley 11.220 del 24/11/94 se inicia en la Provincia de Santa Fe una profunda transformación de los servicios de agua potable y cloacas. El Estado deja de ser el prestador del servicio que efectuaba a través de la DIPOS, pasando a ser a partir del 05-12-95 según el Decreto 3495, el regulador y controlador de la prestación de Ios servicios a través del ENRESS, entidad autárquica con capacidad de derecho público y capital propio que posee el poder de policía en todo el ámbito territorial cualquiera sea el prestador. El servicio se otorgó bajo la forma de concesión de servicios públicos por el término de 30 años.

Es importante observar que si bien la concesión se otorgó por las doce ciudades más importantes de la Provincia en que la DIPOS brindaba el servicio, otras más pequeñas seguían conservando el actual suministro, el que es realizado por municipalidades de segundo orden, comunas, cooperativas o mutuales, estableciéndose la competencia del ENRESS en todo el ámbito provincial, vale decir, que incluye tanto los servicios concesionados como a los demás servicios no concesionados.

Los objetivos perseguidos en la introducción de capitales privados tenían la clara intención de sumar a las nuevas técnicas profesionales de "management", la realización de inversiones postergadas y el manejo de tarifas que se hallaban apartadas del real costo de la prestación del servicio, situación aún no solucionada por el actual prestador del suministro de agua.

\section{3. ÁMBITO, OBJETIVOS, HIPÓTESIS, METODOLOGÍA DE LA INVESTIGACIÓN}

La investigación se ha realizado dentro del territorio de la Provincia de Santa Fe, examinándose los distintos tipos de tarifas que se aplican para los servicios de agua potable y desagües cloacales, independientemente de que el suministro lo realice el prestador de la concesión, es decir, Aguas Provinciales de Santa Fe, como los más de doscientos cincuenta prestadores no concesionados tales como municipalidades, comunas, cooperativas o mutuales.

El objetivo de la investigación es conocer cuales son las causas o factores que determinan las diferencias en los valores de las tarifas en la provisión del servicio entre distintos sectores: Concesionados y no Concesionados, entre los mismos no Concesionados o entre la provisión pública y privada, con la intención de poder trazar un diagnóstico y evaluación -conforme al tiempo y los recursos disponibles-que permita producir conclusiones que estén orientadas para el diseño de políticas públicas que den respuestas a una eficiente regulación económica de los servicios sanitarios.

En el marco de la misma, se plantea como hipótesis general o supuesto que se procura verificar, el que "existe una relación o nexo 
entre los niveles de las tarifas aplicadas y percibidas por los distintos prestadores y su situación demográfica, ubicación geográfica, diferenciación precio-área de concesión y no concesión y elasticidad precio-consumo".

Dentro de este esquema se propone verificar algunas hipótesis específicas u operacionales tales como:

"A mayor dimensión poblacional menor precio de los servicios cobrados por los prestadores":

a) "Ias tarifas de los servicios prestados en los departamentos de la franja Centro-Oeste de la Provincia de Santa Fe presentan valores tarifarios mayores que los prestados sobre la ribera del Paraná";

b) "El cobro de las tarifas de Aguas y Cloacas de los prestadores que están fuera del área concesionada es superior a las cobradas por la Empresa Concesionaria de los servicios sanitarios";

c) "El mayor consumo de agua potable es proporcional al incremento en el nivel de las tarifas".

El diseño metodológico desarrollado consiste en un análisis básicamente exploratorio teniendo en cuenta que la amplitud del mismo dependería de la riqueza de información que los prestadores pudieren brindar, durante la investigación sobre las distintas variables económicas que se ponderan para la aplicación de los cuadros tarifarios. En esa dirección, los lineamientos utilizados fueron el análisis de datos secundarios, la recopilación de datos primarios fruto de la investigación realizada con los prestadores, las que, en la medida de lo posible, fueron cotejada con información perteneciente al Ente de Control Provincial de cuadros tarifarios aprobados por sus máximas autoridades, así como también se realizaron Entrevistas no estructuradas con funcionarios, técnicos y especialistas en distintas materias de ese Organismo.
En las subsiguientes carillas intentaremos develar las hipótesis establecidas, considerando la importancia que representaría la investigación, en el marco temporal en que se realiza, dadas las diversas postergaciones de renegociación de las tarifas de los servicios sanitarios que se vienen produciendo entre el concedente de ellos, es decir, el Gobierno de la Provincia de Santa Fe y su actual concesionario la Empresa Aguas Provinciales de Santa Fe S.A.

\section{CONCLUSIONES}

Luego de la extensa recopilación y análisis de datos, consecuencia del camino metodológico adoptado y de las tareas coordinadas que se fueron plasmando precedentemente, pueden extraerse las siguientes conclusiones:

Existe una marcada relación entre los distintos niveles tarifarios con respecto a su situación poblacional dado una misma aplicación tecnológica, su ubicación geográfica y al área prestacional de los servicios, conforme al cumplimiento de las hipótesis operacionales que se correlacionan con estos tópicos. En cambio no se aprecia un cumplimiento de la hipótesis operacional con respecto a la elasticidad precio consumo.

Preliminarmente, no se han advertido en el estudio-exclusivamente de los prestadores no concesionados- diferencias o respuestas en los niveles tarifarios de los servicios de agua potable ante incrementos poblacionales. En esa línea, se pudo comprobar que para localidades con poblaciones servidas hasta los 7500 habitantes, los valores de los cuadros tarifarios tuvieron un comportamiento inferior al promedio de todos los prestadores. En cambio, en poblaciones con más de 7500 usuarios, sus valores unitarios promedios tuvieron un comportamiento por encima de la media provincial del valor tarifario. La razón de tal situación se ha podido verificar en el cuadro $\mathrm{N}^{\circ} 10$ en el que se han logrado com- 
probar fuertes descensos en los costos en el orden del 30\% como consecuencia de haber excluidos aquellos servicios que operaban con tratamiento de Osmosis Inversas.

Además, dentro de la esfera de servicios suministrados en poblaciones mayores a 7500 , el $74 \%$ es prestado por Cooperativas lo que particularmente observamos en el punto 5.2.2. Dichas prestaciones se encarecían con respecto a los valores de los servicios proveídos en la orbita estatal (comunas o municipalidades) en un $18 \%$ aproximadamente.

La comparación así establecida, teniendo únicamente como parámetro valores tarifarios absolutos, arrojaría conclusiones que despojarían de veracidad a la hipótesis sustentada por el autor en relación a no-cumplimiento de la afirmación de que mayores conjuntos poblacionales provocarían valores tarifarios en descenso, por lo menos en el caso de la prestación del servicio fuera de la órbita de la concesión de los servicios sanitarios. En cambio esta hipótesis se cumpliría, si se extraen de los análisis tarifarios descriptos anteriormente aquellos servicios que suministren los mismos a través de sistemas de Osmosis Inversas, lo cual arrojaría guarismos para las franjas poblacionales mayores a 7.500 habitantes, por debajo de la media tarifaria provincial y de los valores establecidos para las demás bandas analizadas.

Los valores tarifarios explorados en toda la provincia pero particularmente en la franja oeste de la misma están en concordancia con las expresiones científicas desarrolladas por especialistas en calidades de aguas. Tal es el caso de formulaciones como la contenida en la exposición efectuada por el Ing. Hammerly ${ }^{1}$, donde traza una línea imaginaria que parte del meridiano $60^{\circ}$ al norte hasta el meridiano $62^{\circ}$ al sur, en la que manifiesta que las aguas de esa zona son de elevadas salinidad, con alto contenido de cloruro, sulfatos, arsénico y flúor que superan en mucho casos los valores admisibles para ser consideradas potables, caracterizando a la misma por la existencia de aguas subterráneas con acuíferos pobres y altas concentraciones de sales tóxicas, es decir que no cuentas con recursos sostenibles apropiados para satisfacer la demanda, demarcando una posición diametralmente opuesta con la franja este de la Provincia que presenta alta calidad físico-química de sus aguas con alta dilución y autodepuración.

Dicha situación desemboca en que los servicios prestados en esas zonas deben incorporar tratamientos adicionales con controles más complejos, los que se realizan en las propias fuentes subterráneas y se extienden a la entrada del sistema de distribución y en la misma red. A manera de citar algunos de los controles, podemos mencionar análisis químicos (turbiedad, color, olor, sabor, conductividad, nitratos, arsénicos, fluor, hierro, etc), análisis bacteriológicos (determinaciones de distintos tipos de bacterias que puedan encontrarse en el agua) y análisis parasitológicos (comprende de detección de Giardias y criptosporidium. En algunos de ellos se colocaron plantas de osmosis inversas a efectos de posibilitar una mayor potabilización de las aguas recogidas.

La incorporación de mecanismos de control como el agregado de mayores insumos para la mejora en la calidad del agua, plantea un incremento de sus costos, los que se trasladan inevitablemente a las respectivas tarifas.

De lo expresado podemos concluir que se cumple con la segunda de las hipótesis operacionales, dado que los niveles tarifarios de los servicios que se operan en la franja Centro-Oeste de la provincia son mayores a los operados en la franja "este" del territorio provincial.

Se podría sostener para verificar la tercera de las hipótesis que las tarifas no lineales en dos partes, aplicadas con un mismo valor por $\mathrm{m}^{3}$ en toda la provincia (tarifas planas) en el 
Area Concesionada dentro del territorio de la Provincia de Santa Fe por parte de la Empresa Aguas Provinciales de Santa Fe S.A. es una de las más bajas de la provincia, si se tiene en cuenta su cobro medido, es decir si se compara los mismos valores en $\mathrm{m}^{3}$ con los demás servicios. De ésta forma sus valores pueden ser sostenidos en todo el territorio provincial por la incidencia de grandes concentraciones poblacionales como son las ciudades de Rosario y Santa Fe y en menor medida San Lorenzo y Reconquista, las que permiten de alguna forma subsidiar costos incrementales en otras ciudades más pequeñas como Esperanza o Galvez o de similar magnitud pero con costos de potablilización mayores como la ciudad de Rafaela. Estas tarifas están distantes en aproximadamente $80 \%$ tomando $15 \mathrm{~m}^{3}$, del promedio tarifario provincial, lo que colocaría a la concesionaria desde éste punto de vista en una posición claramente ventajosa sobre los demás prestadores a los efectos de demostrar una futura revisión de los aranceles aplicados a sus usuarios. Lo que hace variar este esquema, es el abandono de la estricta medición del agua en sus magnitudes cúbicas, pasando a otras que sean frutos de meras conversiones matemáticas de metros cuadrados (tales son los casi $80 \%$ de los usuarios que no poseen medidores) a dimensiones cúbicas, en cuyo caso consumos presuntos de agua podría estar por encima en promedio en un $86 \%$ de aquellos que poseen mediciones reales y sus valores absolutos ( $\$ 10.76$ ) estría igualados a la media provincial de los prestadores no concesionados.

La insostenibilidad de las tarifas de la concesionaria en comparación con otros prestadores se da en el campo de los servicios de desagües cloacales. Bajo el mismo parámetro descripto anteriormente la concesionaria percibe tales servicios según posean sistema de medición o no -tal situación se verifica en el capítulo $V_{-}$, para aquellos usuarios que posean los correspondiente aparatos de medición de sus consumos las tarifas para $15 \mathrm{~m}^{3}$ rondarían valores que se ubicaría por encima de la media provincial cercanos al $20 \%$, pero en cambio si se tomaran las magnitudes de usuarios que no poseen medidores y que hayan sido transferidos con sistemas de mediciones catastrales desde la ex Dirección Provincial de Obras Sanitarias (80\% aproximadamente) y que de acuerdo a los promedios de metros cuadrados que se tomaron como base para el presente estudio, arrojaban conversiones cúbicas cercanas a los 59.66 $\mathrm{m} 3$ bimestrales, sus diferencias porcentuales tarifarias estarían por encima del promedio provincial alrededor del $120 \%$.

Tal situación nos permite inferir que según como sean tomados las variables a considerar los valores tarifarios operados por la concesionaria en el servicio del agua exclusivamente, en algunos casos son claramente económicos si se tomara la medición estricta del consumo y en otros sus valores se alejarían en comparación con algunos prestadores no concesionados, pero siempre por debajo de la media Provincial. En cambio los operados para los servicios de desagües cloacales su aplicación están levemente por encima de la media provincial en los casos de existencia de mecanismos de medición y superiores (120\%) en los casos de cobro de los servicios por conversión de metros cuadrados a cúbicos por cuentas transferidas desde los servicios brindados por el Estado hacia la actual concesionaria.

La última de las hipótesis a develar está relacionada con los niveles tarifarios y el nivel de consumo. Dado que el agua potable es un elemento vital para la vida de las personas y es un recurso cada vez más escaso, su uso racional tal cual lo marca la Ley 11.220 debe entenderse de dos maneras. La primera de ellas es la propensión al consumo medido, esto equivale a decir que los servicios que 
operan el suministro del agua tienen y deben tener la obligación de canalizar partes de sus recursos en inversiones que posibiliten la mayor cantidad de colocaciones de artefactos que controlen el suministro del vital líquido. Por otra parte, el castigo de grandes consumos de agua lleva a un uso racional del agua potable.

En esa línea de investigación se concluye que los mayores consumos de agua no se condicen con valores tarifarios proporcionalmente iguales a dicha variación. Así si al promedio provincial para consumos de $15 \mathrm{~m}^{3}$ con un precio $\$ 11,30$ se lo incrementara en un $50 \%$, es decir surgiría una cantidad de 22,5 $\mathrm{m}^{3}$ el valor tarifario se incrementa un $43 \%$. Si los metros cúbicos a considerar fueran 30 $\mathrm{m}^{3}$ (incrementaríamos un 100\% la cantidad de agua suministrada) el incremento de las tarifas se modificaría en un $87 \%$. Esto equivale a decir que se debería diseñar mecanismos urgentes de protección del consumo excesivo de agua para evitar abusos desmedidos, sobre todo en algunas zonas que su obtención se dificulta enormemente.

Por último es importante remarcar que la regulación tarifaria que ejerce el ENRESS sobre la empresa concesionada es mixta, con precios tope y ajuste en función de índice de costos y cumplimiento de inversiones por expansión (control en el cumplimiento de los Planes de Mejora y Desarrollo). En cambio las tarifas que se fijen sobre las prestaciones brindadas fuera del Área Concesionada serán decididas por las autoridades municipales o comunales con sujeción a los principios comentados en el punto 4.7.y a las respectivas normativas locales.

Sin perjuicio de tocar aspectos que de- berían ser cubiertos por futuras líneas de investigación, es preciso remarcar que el Ente Regulador de Servicios Sanitarios presenta determinados déficit de capacidad institucional para poder alcanzar a controlar todos los parámetros en materia de tarifas fundamentalmente en lo que respecta a los prestadores no concesionarios.

De allí, dada la necesidad y oportunidad de abrir un debate sobre lo que se necesita en materia de regulación y como lograrlo, es que consideramos que el ente debe mejorar sustancialmente la capacidad de control de las tarifas cualquiera sea el prestador, exigiendo severamente el cumplimiento de la información a la que están obligados los distintos servicios y adoptar un sistema formular de tarifas en la que se vayan incorporando determinadas variables como cantidad de población, tipo de tratamiento, parámetros de calidad del agua a captar, números de conexiones, superficie del servicio a cubrir, tasa de rentabilidad, etc. De ésta manera se podrá conseguir un resultado tarifario en línea con las variables aplicadas.

Asimismo, se debería apoyar decididamente la construcción de estructuras regulatorias Nación-Provincia que en forma incipiente se está verificando en estudios desarrollados por la Federación de Entes Reguladores, en la que se manejen informaciones de tarifas y otras variables y las mismas sean compartidas por todos los Organismo de Control de Servicios Sanitarios del país, de manera tal que se pueda lograr una robusta y completa base de datos en la que se puedan comparar los cuadros tarifarios de estos servicios en los distintos estados subnacionales.

\section{NOTA}

${ }^{1}$ Véase Hammerly, Jorge (2002): Curso sobre la regulación de los servicios sanitarios-prestadores no concesionados. 


\section{BIBLIOGRAFÍA}

Asensio, Miguel Angel (1995): "Monopolios naturales y políticas públicas", CECYT (FACPCE). Informe $N^{\circ} 5$, Buenos Aires.

Asensio, Miguel Angel (2000): Federalismo fiscal. Fundamentos. Análisis comparado y el caso argentino, Ed. Ciudad Argentina, Universidad del Salvador, Buenos Aires.

Azpiazu, Daniel y Basulado, Eduardo (1999): "Regulación de los Servicios Públicos y concentración económica. Hacia el Plan Fénix: diagnósticos y propuestas”, Revista Enoikos № 19, Facultad de Ciencias Económicas, Universidad de Buenos Aires.

Barrionuevo, A. y Lahera, E. (1998): ¿Qué hay de nuevo en la regulaciones? Telecomunicaciones, electricidad y agua en América Latina - Política de Regulación, Ed. Eudeba y CLAD, Buenos Aires.

Bianchi, Alberto B. (2001): La regulación económica. Desarrollo histórico. Régimen jurídico de los Entes Reguladores en la Argentina, Ed. Desalma, Buenos Aires.

Canasi, José (1974): Derecho administrativo, Vol. II, Buenos Aires.

Cicero, Nidia Karina (1996): Senvicios Públicos: control y protección, Ed. Ciudad Argentina, Colección Monografías Jurídicas, Buenos Aires.

Cincunegui, Juan Bautista (1994): “Regulación pública”, Revista Argentina del Régimen de la Administración Pública (RAP) № 184, Ed. Ciencias de la Administración S.R.L., Buenos Aires.

Colomé, R.A.: Microeconomía introductoria, Volumen I, Córdoba.

Correa Bau, Carlos (2001): Empresa modelo, Benchmarking y Costo de capital, Seminario de AFERAS, Buenos Aires.

FIEL (Fundación de Investigaciones Económicas Latinoamericanas): La Regulación de la competencia y de los Servicios Públicos: teoría y experiencia Argentina reciente, Buenos Aires, 1998.

Ghersi, Carlos A.: Derechos y responsabilidades de la empresas y consumidores ed. Organizaciones Mora Libros.

Giandomenico Majone y Antonio La Spina (1993): El Estado regulador, Gestión y Política Pública, C.I.D.E, Vol. II, México.

Haveman, Robert H. (1970): El Sector Público, Centro Regional de Ayuda Técnica, México.

Jones, Leroy (1994): Appropriate Reulatory Technology: The Interplay of Economic and Institutional Conditions, Proceedings of the World Bank Annual Conference on Development Economics. 1993, Michael Bruno y Boris Pleskovic (comps.), Banco Mundial, Washington, D.C. 
Jouravlev, Andrei (2001): Regulación de la industria de agua potable, volumen I: necesidades de información y regulación estructural, recursos naturales e infraestructura, CEPAL, Santiago de Chile, Chile.

Kessides, Christine (1993): The Institutional Options for the Provision of Insfraestructure, World Bank Discussion Papers, № 213.

Mitnick, B.M. (1980): The Political Economy of Regulation, Columbia University Press. Nueva York.

Mochón, Francisco y Beker, Victor: Economía: principios y aplicaciones, segunda edición, Editorial Mc Graw Hill. Madrid, España, 1997.

Nallar, Daniel M. (1999): El Estado regulador y el nuevo mercado del Senvicio Público. Análisis jurídico sobre la privatización, la regulación y los entes reguladores, Ed. Depalma, Argentina. Buenos Aires.

Oszlak, Oscar y Felder, Ruth (1999): La capacidad de regulación estatal en la Argentina. Quis Custodiel Custodes? La Argentina que viene. Buenos Aires.

Rees, Judith (1998): Regulation and Private Participation in the water and Sanitation Sector, Natural Resources Forum, vol. 22, № 2.

Remes Lenicov: El papel del Estado en los Servicios Públicos Privatizados. Diario la Nación 25/04/99.

Samuelson, Paul y Nordhaus, William (1995): Economía, Mac Graw-Hill, Madrid.

Sarmiento García, Jorge (1994): Los Servicios Públicos: régimen jurídico actual, Ed. Depalma, Buenos Aires Argentina.

Sarmiento García, Jorge (1999): Concesión de Servicios Públicos, Ed. Ciudad Argentina, Buenos Aires.

Selznick, P. (1985): Focusing Organizational Research on Regulation, Noll.

Universidad Argentina de la Empresa (2002): Principios tarifarios y mecanismos regulatorios para los Servicios Públicos, Centro de Estudios Económicos de la Regulación, Buenos Aires.

Urbiztondo, Santiago (1993): Hacia una teoría positiva sobre el objetivo de las privatizaciones. Argentina.

$6^{\circ}$ Informe Anual remitido al ENRESS por la actual Concesionaria Agua Provinciales de Santa Fe por el período 01-01-2001 al 31-12-2001. 
